This item was submitted to Loughborough's Research Repository by the author.

Items in Figshare are protected by copyright, with all rights reserved, unless otherwise indicated.

\title{
Harmonic moments and an inverse problem for the heat equation
}

PLEASE CITE THE PUBLISHED VERSION

LICENCE

CC BY-NC-ND 4.0

\section{REPOSITORY RECORD}

Kawashita, M., Y.V. Kurylev, and H. Soga. 2019. "Harmonic Moments and an Inverse Problem for the Heat Equation". figshare. https://hdl.handle.net/2134/846. 


\title{
HARMONIC MOMENTS AND AN INVERSE PROBLEMS FOR THE HEAT EQUATION
}

\author{
By Mishio KAWASHITA, Yaroslav KURYLEV and Hideo SOGA
}

\section{$\S 1$. Introduction}

The paper is devoted to the solution of the inverse boundary problem for the heat equation. Let $\Omega$ be a connected bounded domain in $\mathbf{R}^{n}(n \geq 2)$ with $C^{l}(l \geq 2)$ boundary $\Gamma$. Consider the mixed problem for the heat equation

$$
\begin{cases}\left(\rho(x) \partial_{t}-\triangle\right) u^{f}(t, x)=0 & \text { in } \quad(0,+\infty) \times \Omega, \\ u^{f}\left(t, x^{\prime}\right)=f\left(t, x^{\prime}\right) & \text { on }(0,+\infty) \times \Gamma, \\ u^{f}(0, x)=0 & \text { on } \Omega .\end{cases}
$$

The density $\rho(x)$ is a $C^{l+\sigma}, 0<\sigma<1$, function on $\bar{\Omega}$ satisfying

$$
0<\rho_{1} \leq \rho(x) \leq \rho_{2}(<+\infty) .
$$

The inverse data used in the paper is a set of normal derivatives $\left.\frac{\partial u^{p}}{\partial \nu}\right|_{(0,2) \times \Gamma}$ where $u^{p}$ is the solution of (1.1) with

$$
f\left(t, x^{\prime}\right)=\chi(t) p\left(x^{\prime}\right) .
$$

Here $\chi(t)$ is a (arbitrary) fixed $C^{\infty}$ function satisfying $0 \leq \chi(t) \leq 1$ in $\mathbf{R}$, $\chi(t)=1$ for $t \geq 1$ and $\chi(t)=0$ for $t \leq 1 / 2$. The function $p\left(x^{\prime}\right)$ in (1.1) is the boundary value of a harmonic polynomial $p(x)$ (i.e. $\triangle p=0$ ).

We assume that $\left.\frac{\partial u^{p}}{\partial \nu}\right|_{(0,2) \times \Gamma}$ or, more precisely,

$$
\int_{\Gamma} \int_{0}^{t} \frac{\partial u^{p}}{\partial \nu}\left(s, x^{\prime}\right) q\left(x^{\prime}\right) d x^{\prime} d s, \quad 0<t<2,
$$

are given for all sources $f$ of form (1.3) with $p \in H P^{m}$, where

$$
H P^{m}=\{\text { harmonic polynomial of degree } \leq m\} \quad(m=0,1,2, \cdots),
$$


and all $q \in H P^{m}$.

In the paper we describe algorithms for an approximate reconstruction of $\rho$ given approximate integrals (1.4) with $p, q \in H P^{m}, m=0,1,2, \ldots$. The algorithms lead to explicit formulae for an approximate solution of the inverse problem under consideration together with an error estimate in the corresponding reconstruction procedure. These error estimates depend upon the parameter $m$ and a "measurement" error, i.e. an error in the inverse data (1.4) and are of the logarithmic character. The algorithms are described in $\S 3$ where we do not use a pseudoanalytic continuation of the inverse data and in $\S 4$ where we utilize results on pseudoanalytic continuation (see e.g. [Car, L]). The corresponding formulae together with the error estimate are given in Theorems 3.4 and 4.4 .

The method used in the paper is the parabolic analog of the Moments' Method introduced in [K-S] for the solution of the inverse boundary spectral problem for the acoustic operator $A_{\rho}=-\rho^{-1} \triangle$. Its main idea is to utilise the generating properties of the products of harmonic polynomials. These polynomials belong to the null-space of the acoustic operator. The considered generating properties are, in fact, an algebraic version of the well-known fact (see e.g. [Cal]) that the linear combinations of the products of harmonic functions are dense in $L^{2}(\Omega)$.

This fact was extensively used for solving inverse boundary problems (see $[\mathrm{S}-\mathrm{U}]$ for the pioneering work in this direction). In the parabolic case the study of the inverse problems for the system (1.1) and even for some more general parabolic equations was carried out by a number of authors. The main results dealt with the question of uniqueness and stability in the identification of the unknown coefficient(s) via various sets of the inverse data on the boundary. A very good introduction to this area together with a number of advanced results is given in [Is; Ch. 9]. However, we would like to stress that our main goal is not to obtain stability estimates pe se but to develop some reconstruction procedures and to obtain stability estimates for these procedures. In its turn such procedures may prove useful for the numerical solution of inverse boundary problems. Indeed, the acoustic variant of the Moments' Method was successfully used in $[\mathrm{K}-\mathrm{P}]$ for the numerical solution of some model inverse problems.

\section{$\S 2$. The direct problem}

Consider the mixed problem (1.1) and denote by $u^{p}(t, x)$ its solution with the source $f$ of form (1.3). When $p, q \in H P^{\infty}\left(=\cup_{m=0}^{\infty} H P^{m}\right)$ we define $\Phi_{\rho}(t ; p, q)$ by

$$
\Phi_{\rho}(t ; p, q)=\int_{\Omega} \rho(x) u^{p}(t, x) \overline{q(x)} d x .
$$


We define also the harmonic moments $M_{\rho}(p, q)$ corresponding to $\rho$ :

$$
M_{\rho}(p, q)=\int_{\Omega} \rho(x) p(x) \overline{q(x)} d x .
$$

In this section we discuss some properties of $\Phi_{\rho}(t ; p, q)$ and relations between $\Phi_{\rho}(t ; p, q), M_{\rho}(p, q)$ and the response operator

$$
R_{\rho}:\left.p\left(x^{\prime}\right) \mapsto \frac{\partial u}{\partial \nu}\right|_{(0,+\infty) \times \Gamma}
$$

Theorem 2.1. $\Phi_{\rho}(t ; p, q)$ is a bilinear mapping from $H P^{\infty} \times H P^{\infty} \rightarrow$ $C^{0}\left(\overline{\mathbf{R}_{+}}\right)$with the following properties:

$$
\begin{aligned}
& \left\|\Phi_{\rho}(\cdot ; p, q)\right\|_{C^{0}\left(\overline{\mathbf{R}_{+}}\right)} \leq C\|p\|_{L^{2}(\Omega)}\|q\|_{L^{2}(\Omega)} \\
& \left\|\Phi_{\rho}(\cdot ; p, q)-\Phi_{\tilde{\rho}(\cdot ; p, q)}\right\|_{C^{0}\left(\overline{\mathbf{R}_{+}}\right)} \\
& \quad \leq C\|\rho-\tilde{\rho}\|_{C^{0}(\bar{\Omega})}\|p\|_{L^{2}(\Omega)}\|q\|_{L^{2}(\Omega)} \\
& \left|\Phi_{\rho}(\cdot ; p, q)-M_{\rho}(p, q)\right| \\
& \leq C e^{-C^{\prime} \lambda_{0} t}\|p\|_{L^{2}(\Omega)}\|q\|_{L^{2}(\Omega)},
\end{aligned}
$$

where $C, C^{\prime}$ are positive constants determined from $\rho_{1}, \rho_{2}$ and $\lambda_{0}$ is the first eigenvalue of the Dirichlet problem for $-\triangle$ on $\Omega$.

Note. We denote by $C, C^{\prime}$ different constants which depend upon $\Omega, \rho_{1}$ and $\rho_{2}$. In the case of their dependence upon some other parameters we note this dependence explicitly.

Proof. Consider the mixed problem

$$
\begin{cases}\partial_{t} w(t, x)-\rho^{-1} \triangle w(t, x)=0 & \text { in }(0,+\infty) \times \Omega, \\ w\left(t, x^{\prime}\right)=0 & \text { on }(0,+\infty) \times \Gamma, \\ w(0, x)=f(x) & \text { on } \Omega,\end{cases}
$$

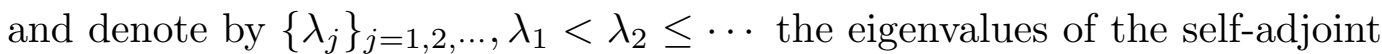
differential operator $-\rho^{-1} \triangle$ with the domain $H^{2}(\Omega) \cap H_{0}^{1}(\Omega)$ in the Hilbert space $L_{\rho}^{2}(\Omega)$ with the inner product $(f, g)_{\rho}=\int_{\Omega} \rho(x) f(x) \overline{g(x)} d x$. We can express the solution $w(t, \cdot)=E(t) f$ in the form

$$
E(t) f=\sum_{j=1}^{\infty} e^{-\lambda_{j} t}\left(f, \varphi_{j}\right)_{\rho} \varphi_{j}
$$


where the functions $\left\{\varphi_{j}\right\}_{j=1,2, \ldots}$ are the $L_{\rho}^{2}$-orthonormal eigenfunctions corresponding to the eigenvalues $\lambda_{j}$. Note that the first eigenvalue $\lambda_{1}$ is estimated by $\lambda_{0}$, i.e. $\lambda_{0} \rho_{2}^{-1} \leq \lambda_{1} \leq \lambda_{0} \rho_{1}^{-1}$.

Then we have

$$
u^{p}(t, x)-\chi(t) p(x)=\int_{0}^{t} E(t-s)\left(-\chi^{\prime}(s) p(x)\right) d s .
$$

But $L_{\rho}^{2}(\Omega)=L^{2}(\Omega)$ as a set and $\rho_{1}\|f\|^{2} \leq\|f\|_{\rho}^{2} \leq \rho_{2}\|f\|^{2}$, where we denote by $\|f\|$ the usual $L^{2}$-norm of $f$. Hence (2.3) yields that

$$
\left\|\int_{0}^{t} E(t-s)\left(-\chi^{\prime}(s) p(x)\right) d s\right\| \leq\left(\rho_{2} / \rho_{1}\right)^{1 / 2} e^{\lambda_{1}(1-t)} \sup _{s \in \mathbf{R}}\left|\chi^{\prime}(s)\right|\|p\| .
$$

Thus, we obtain

$$
\begin{aligned}
& \left|\Phi_{\rho}(t ; p, q)-\int_{\Omega} \rho(x) p(x) \overline{q(x)} d x\right| \\
& \quad \leq\left|(1-\chi(t)) \int_{\Omega} \rho p \bar{q} d x\right|+\left|\int_{\Omega} \rho(u-\chi p) \bar{q} d x\right| \\
& \quad \leq\left(e^{\lambda_{1}} \rho_{2}+\left(\rho_{2} / \rho_{1}\right)^{1 / 2} e^{\lambda_{1}}\left\|\chi^{\prime}\right\|_{C^{0}\left(\overline{\mathbf{R}_{+}}\right)}\right) e^{-\lambda_{1} t}\|p\|\|q\|,
\end{aligned}
$$

which proves (i) and (iii).

Denote by $\tilde{u}^{p}(t, x)$ the solution of the equation (1.1) with $\tilde{\rho}(x)$ instead of $\rho(x)$, and by $\widetilde{E}(t)$ the operator of form (2.3) corresponding to $\tilde{\rho}$. We see that

$$
u^{p}(t, x)-\tilde{u}^{p}(t, x)=\int_{0}^{t} E(t-s)\left\{(\tilde{\rho}-\rho) \rho^{-1} \partial_{s} \tilde{u}^{p}(s, \cdot)\right\} d s .
$$

Therefore (1.2) implies that

$$
\left\|u^{p}(t, x)-\tilde{u}^{p}(t, x)\right\| \leq \rho_{1}^{-1}\left(\rho_{2} / \rho_{1}\right)^{1 / 2}\|\rho-\tilde{\rho}\|_{C^{0}(\bar{\Omega})} \int_{0}^{t}\left\|\partial_{s} \tilde{u}(s, \cdot)\right\| d s .
$$

From (2.4) it follows that $\partial_{s} \tilde{u}^{p}(s, \cdot)=-\int_{0}^{s} \partial_{t} \widetilde{E}(s-t)\left[\chi^{\prime}(t) p\right] d t=\chi^{\prime}(s) p-$ $\int_{0}^{s} \widetilde{E}(s-t) \chi^{\prime \prime}(t) p d t$, which yields that

$$
\begin{aligned}
& \left\|\partial_{s} \tilde{u}^{p}(s, \cdot)\right\| \leq\left|\chi^{\prime}(s)\right|\|p\| \\
& \qquad+\left(\rho_{2} / \rho_{1}\right)^{1 / 2} \int_{0}^{1} e^{-\tilde{\lambda}_{1}(s-t)} d t\left\|\chi^{\prime \prime}\right\|_{C^{0}\left(\overline{\mathbf{R}_{+}}\right)}\|p\| .
\end{aligned}
$$

In the above, $\tilde{\lambda}_{1}$ is the first eigenvalue of the self-adjoint realization of $-\tilde{\rho}^{-1} \triangle$ on $L_{\tilde{\rho}}^{2}(\Omega)$. Combining this with $(2.5)$, we obtain

$$
\begin{aligned}
\left|\Phi_{\rho}(t ; p, q)-\Phi_{\tilde{\rho}}(t ; p, q)\right| & \leq\left|\int_{\Omega}(\tilde{\rho}-\rho) u(t, \cdot) \bar{q} d x\right| \\
& +\left|\int_{\Omega} \tilde{\rho}\{u(t, \cdot)-\tilde{u}(t, \cdot)\} \bar{q} d x\right| \leq C\|\rho-\tilde{\rho}\|_{C^{0}(\bar{\Omega})}\|p\|\|q\| .
\end{aligned}
$$

This completes the proof of Theorem 2.1.

The following theorem shows the relationship between the form $\Phi_{\rho}(t ; p, q)$ and the response operator $R_{\rho}$ : 
Lemma 2.2. For any $p, q \in H P^{\infty}$ we have

$$
\begin{aligned}
\Phi_{\rho}(t ; p, q)=\int_{\Gamma} \int_{0}^{t} R_{\rho}\left[\left.\chi p\right|_{\Gamma}\right]\left(s, x^{\prime}\right) d s \overline{q\left(x^{\prime}\right)} d x^{\prime} \\
\quad-\int_{0}^{t} \chi(s) d s \int_{\Gamma} p\left(x^{\prime}\right) \frac{\partial \overline{q\left(x^{\prime}\right)}}{\partial \nu} d x^{\prime} .
\end{aligned}
$$

Proof. By integration by parts we have

$$
\begin{aligned}
\int_{\Omega} \int_{0}^{t}\left\{\left(\partial_{s}-\rho^{-1} \triangle\right) u^{p}(s, x)\right\} \overline{q(x)} \rho(x) d s d x \\
=\int_{\Omega} u^{p}(t, x) \overline{q(x)} \rho(x) d x-\int_{\Omega} u^{p}(0, x) \overline{q(x)} \rho(x) d x \\
\quad-\int_{\Omega} \int_{0}^{t} u^{p}(s, x) \overline{\triangle q(x)} d s d x \\
\quad-\int_{\Gamma} \int_{0}^{t} \frac{\partial u^{p}}{\partial \nu}\left(s, x^{\prime}\right) \overline{q\left(x^{\prime}\right)} d s d x^{\prime}+\int_{\Gamma} \int_{0}^{t} u^{p}\left(s, x^{\prime}\right) \overline{\frac{\partial q}{\partial \nu}\left(x^{\prime}\right)} d s d x^{\prime} .
\end{aligned}
$$

As $u^{p}$ is a solution of (1.1) and $q \in H P^{\infty}$ the above inequality implies that

$$
\begin{aligned}
0=\Phi_{\rho}(t ; p, q) & -\int_{\Gamma} \int_{0}^{t} R_{\rho}\left[\left.\chi p\right|_{\Gamma}\right]\left(s, x^{\prime}\right) \overline{q\left(x^{\prime}\right)} d s d x^{\prime} \\
& +\int_{\Gamma} \int_{0}^{t} \chi(s) d s p\left(x^{\prime}\right) \overline{\frac{\partial q}{\partial \nu}\left(x^{\prime}\right)} d x^{\prime}
\end{aligned}
$$

This proves Lemma 2.2.

In the inverse problems, generally, we expect recovering $\rho$ by the measurements expressed in terms of the responce operator. Lemma 2.2 implies that our original setting of the inverse problem may be reduced to the inversion of the mapping : $\rho \mapsto \Phi_{\rho}$.

We start with verifying the uniqueness of the inverse problem.

Theorem 2.3. If for any $p, q \in H P^{\infty}, \Phi_{\rho}(t ; p, q)$ is equal to $\Phi_{\tilde{\rho}}(t ; p, q)$ on an interval $(1<) a<t<b$, then $\rho$ coinsides with $\tilde{\rho}$.

Remark. Lemma 2.2 means that $\Phi_{\rho}=\Phi_{\tilde{\rho}}$ if $R_{\rho}=R_{\tilde{\rho}}$, and therefore the unique determination of $\rho$ by $R_{\rho}$ is derived from Theorem 2.3.

Proof of Theorem 2.3 is based on the following lemma. 
Lemma 2.4. Any polynomial can be expressed as a linear conbination of products of the harmonic polynomials.

For the proof of Lemma 2.4, see [K-S; Proposition 3].

Proof of Theorem 2.3. The solution $u^{p}(t, x)$ in (1.1) becomes analytic in $t$ for $t>1$ (cf. (2.3)). Therefore, if $\Phi_{\rho}(t ; p, q)=\Phi_{\tilde{\rho}}(t ; p, q)$ on $(a, b), \Phi_{\rho}(t ; p, q)$ is equal to $\Phi_{\tilde{\rho}}(t ; p, q)$ on $(1,+\infty)$. Hence, by (iii) of Theorem 2.1 , we have

$$
M_{\rho}(p, q)=\lim _{t \rightarrow \infty} \Phi_{\rho}(t ; p, q)=\lim _{t \rightarrow \infty} \Phi_{\tilde{\rho}}(t ; p, q)=M_{\tilde{\rho}}(p, q) ; \quad p, q \in H P^{\infty} .
$$

By Lemma 2.4 this implies that

$$
\int_{\Omega} \rho(x) x^{\alpha} d x=\int_{\Omega} \tilde{\rho}(x) x^{\alpha} d x
$$

for any multi-index $\alpha$. As the domain $\Omega$ is bounded this implies that $\rho=\tilde{\rho}$. The proof is complete.

\section{$\S 3$. Reconstruction of $\rho(x)$}

In this section, we reconstruct $\rho(x)$ approximately, by employing the harmonic moments $M_{\rho}(p, q)$ with $p, q \in H P^{m}$ where $m$ is a sufficiently large positive integer.

The reconstruction is based on the fact that the Gaussian distribution

$$
(\sqrt{\pi})^{-n} \mu^{n} \exp \left(-\mu^{2}|x|^{2}\right)=(\sqrt{\pi})^{-n} \mu^{n} \sum_{k=0}^{\infty} \frac{1}{k !}\left(-\mu^{2}|x|^{2}\right)^{k}
$$

tends to the Dirac $\delta$-function as $\mu \rightarrow+\infty$ (note that $\int_{\mathbf{R}^{n}} \mu^{n} \exp \left(-\mu^{2}|y|^{2}\right) d y$ $=(\sqrt{\pi})^{n}$ for any $\left.\mu>0\right)$. Namely, we use

Lemma 3.1. Let

$$
\delta_{\mu}^{m}(x)=(\sqrt{\pi})^{-n} \mu^{n} \sum_{k=0}^{m / 2} \frac{\left(-\mu^{2}|x|^{2}\right)^{k}}{k !}
$$

where $\mu \geq 1$ and $m$ ia a positive even integer. Then for any $\rho(x) \in C^{l+\sigma}(\bar{\Omega})$ $(0 \leq l \leq m / 2$, integer, $0<\sigma<1)$ we have

$$
\begin{aligned}
\| \rho(x)-\int_{\Omega} \delta_{\mu}^{m} & (x-y) \rho(y) d y \|_{C^{l}\left(\mu^{-1 / 2}\right)} \\
& \leq C\left\{\|\rho\|_{C^{l+\sigma}} \mu^{-\sigma / 2}+\left(C^{\prime} \mu\right)^{m} m^{-m / 2+l}\right\},
\end{aligned}
$$


where $C, C^{\prime}$ are independent of $\rho, \mu$ and $m$. Here we denote by $\|\rho\|_{C^{l+\sigma}}$ the $C^{l+\sigma_{-n o r m}}$ of $\rho$ in $\bar{\Omega}$ and by $\|\rho\|_{C^{l}(\epsilon)}$ - the $C^{l}$-norm of $\rho$ in $\overline{\Omega_{\epsilon}}$. In its turn, $\Omega_{\epsilon}=\{x \in \Omega \mid \operatorname{dist}(x, \Gamma)>\epsilon\}$.

Proof. For $f \in C^{l}(\bar{\Omega})$ we denote by $D f$ the $C^{l}$-continuation of $f$ onto $\mathbf{R}^{n}$ with $\operatorname{supp}[D f] \subset\{x \mid \operatorname{dist}(x, \Omega)<1\}$ and $\|D f\|_{C^{l}\left(\mathbf{R}^{n}\right)} \leq 2\|f\|_{C^{l}}$. As $\int_{\mathbf{R}^{n}} \delta_{\mu}^{\infty}(x) d x=1$, then for any $|\alpha| \leq l$

$$
\begin{aligned}
\partial_{x}^{\alpha} \rho(x)-\partial_{x}^{\alpha} \int_{\Omega} \delta_{\mu}^{m}(x-y) \rho(y) d y & =\int_{\mathbf{R}^{n}} \delta_{\mu}^{\infty}(y)\left(\partial_{x}^{\alpha} \rho(x)-\partial_{x}^{\alpha} D \rho(x-y)\right) d y \\
+\int_{\mathbf{R}^{n} \backslash \Omega} \partial_{x}^{\alpha} \delta_{\mu}^{\infty}(x-y) D \rho(y) d y & +\int_{\Omega}\left(\partial_{x}^{\alpha} \delta_{\mu}^{\infty}(x-y)-\partial_{x}^{\alpha} \delta_{\mu}^{m}(x-y)\right) \rho(y) d y \\
& =I_{1}+I_{2}+I_{3} .
\end{aligned}
$$

Since $\delta_{\mu}^{\infty}(x)-\delta_{\mu}^{m}(x)=(\sqrt{\pi})^{-n} \mu^{n} R_{m}\left(\mu^{2}|x|^{2}\right)$, where

$$
R_{m}(X)=\frac{X^{m / 2+1}}{(m / 2) !} \int_{0}^{1}(1-\theta)^{m / 2+1} e^{-\theta X} d \theta
$$

then for $|\alpha| \leq m / 2+1$ we obtain

$$
\left|\partial_{x}^{\alpha}\left(\delta_{\mu}^{\infty}(x)-\delta_{\mu}^{m}(x)\right)\right| \leq \frac{4^{|\alpha|}|\alpha| !}{(m / 2+1-|\alpha|) !}(\sqrt{\pi})^{-n} \mu^{n+|\alpha|}(\mu|x|)^{2 m+1-|\alpha|} .
$$

This implies that

$$
\begin{aligned}
\left|I_{3}\right| & \leq(\sqrt{\pi})^{-n} \mu^{n+|\alpha|} \frac{4^{|\alpha|}|\alpha| !}{(m / 2+1-|\alpha|) !}\left(\mu r_{0}\right)^{2 m+1-|\alpha|} \int_{\Omega} \rho(y) d y \\
& \leq(\sqrt{\pi})^{-n} 4^{|\alpha|}|\alpha| ! \mu^{n} \frac{(m / 2)^{|\alpha|}}{r_{0}^{|\alpha|}} \frac{\left(\mu r_{0}\right)^{(2 m+1)}}{(m / 2) !} \int_{\Omega} \rho(y) d y \\
& \leq(\sqrt{\pi})^{-n} r_{0}^{2}|\alpha| ! \mu^{n+m+2}\left(\frac{2 m}{r_{0}}\right)^{|\alpha|} \frac{\left(2 e n r_{0}^{2}\right)^{m / 2}}{m^{m / 2}} \int_{\Omega} \rho(y) d y,
\end{aligned}
$$

where $r_{0}=\operatorname{diam}(\Omega)$ and we use Stirling formula to estimate $\left(\frac{m}{2}\right)$ ! for sufficiently large $m$.

Since $|x-y| \geq \mu^{-1 / 2}$ holds if $x \in \Omega_{\mu^{-1 / 2}}$ and $y \in \mathbf{R}^{n} \backslash \Omega$, we obtain

$$
\begin{aligned}
\left|I_{2}\right| & \leq C\|\rho\|_{C^{0}} \int_{\mathbf{R}^{n} \backslash \Omega} \mu^{n+|\alpha|}(1+\mu|x-y|)^{|\alpha|} e^{-\mu^{2}|x-y|^{2}} d y \\
& \leq C\|\rho\|_{C^{0}} \mu^{|\alpha|} e^{-\mu / 2} \int_{\mathbf{R}^{n}}(1+|y|)^{|\alpha|} e^{-|y|^{2}} d y
\end{aligned}
$$


At last

$$
\begin{aligned}
\left|I_{1}\right| \leq \int_{|y| \leq \mu^{-1 / 2}} \delta_{\mu}^{\infty}(y)\|\rho\|_{C^{l+\sigma}}|y|^{\sigma} d y & \\
& +2 \int_{|y|>\mu^{-1 / 2}} \delta_{\mu}^{\infty}(y)\|D \rho\|_{C^{l}\left(\mathbf{R}^{n}\right)} d y \\
& \leq C\left(\mu^{-\sigma / 2}\|\rho\|_{C^{l+\sigma}}+e^{-\mu} \int_{\mathbf{R}^{n}} e^{-|y|^{2}} d y\|\rho\|_{C^{l}}\right)
\end{aligned}
$$

Combining the above estimates we obtain Lemma 3.1.

The Proof is complete.

We fix a complete system $\left\{p_{i}^{m}\right\}_{i=1, \cdots, N(m)}$ in $H P^{m}$ so that $\left\{p_{i}^{m}\right\} \subset$ $\left\{p_{i}^{m+1}\right\}$. Lemma 2.4 means that any $x^{\alpha}(|\alpha| \leq m)$ is expressed in the form

$$
x^{\alpha}=\sum_{i, j=1}^{N(m)} C_{i, j}^{\alpha} p_{i}^{m}(x) \overline{p_{j}^{m}(x)} .
$$

The function $\delta_{\mu}^{m}(x-y)$ in Lemma 3.1 is then decomposed into a sum of polynomials $x^{\alpha} y^{\beta}$ :

$$
\delta_{\mu}^{m}(x-y)=\sum_{|\alpha+\beta| \leq m} C_{m, \alpha, \beta} \mu^{n+|\alpha+\beta|} x^{\alpha} y^{\beta}
$$

Therefore the integral $\int_{\Omega} \delta_{\mu}^{m}(x-y) \rho(y) d y$ is then represented in terms of the harmonic moments $M_{\rho}(p, q)$

$$
\int_{\Omega} \delta_{\mu}^{m}(x-y) \rho(y) d y=\sum_{|\alpha+\beta| \leq m} C_{m, \alpha, \beta} \mu^{n+|\alpha+\beta|} x^{\alpha} \sum_{i, j=1}^{N(m)} C_{i j}^{\beta} M_{\rho}\left(p_{i}^{m}, p_{j}^{m}\right)
$$

This representation together with Lemma 3.1 imply the possibility of an approximate reconstruction of $\rho$ in terms of the harmonic moments. For this end we introduce the polynomial $Q_{\mu}^{m}(x ; M)$ where $M$ is a bilinear form on $H P^{\infty} \times H P^{\infty}$ :

$$
Q_{\mu}^{m}(x ; M)=\sum_{|\alpha+\beta| \leq m} C_{m, \alpha, \beta} \mu^{n+|\alpha+\beta|} x^{\alpha} \sum_{i, j=1}^{N(m)} C_{i j}^{\beta} M\left(p_{i}^{m}, p_{j}^{m}\right)
$$


Theorem 3.2. (i) The mapping : $M \mapsto Q_{\mu}^{m}(x ; M)$ is continuous in the following sense:

$$
\begin{gathered}
\left\|Q_{\mu}^{m}(x ; M)-Q_{\mu}^{m}(x ; \widetilde{M})\right\|_{C^{l}} \\
\leq C \mu^{n} e^{C^{\prime} \mu^{2}}\|M-\widetilde{M}\|_{m} \max _{|\beta| \leq m} \sum_{i, j=1}^{N(m)}\left|C_{i j}^{\beta}\right|\left\|p_{i}^{m}\right\|\left\|p_{j}^{m}\right\|,
\end{gathered}
$$

where

$$
\|M-\widetilde{M}\|_{m}=\sup \left\{\frac{|M(p, q)-\widetilde{M}(p, q)|}{\|p\|\|q\|} ; \quad p, q \in H P^{m}\right\}
$$

(ii) Let $\rho(x) \in C^{l+\sigma}(\bar{\Omega})(0 \leq l \leq m / 2$, integer $0<\sigma<1)$. Then we have

$$
\begin{aligned}
\| \rho(x)- & Q_{\mu}^{m}\left(x, M_{\rho}\right) \|_{C^{l}} \\
& \leq C_{l}\left\{\|\rho\|_{C^{l+\sigma}} \mu^{-\sigma / 2}+\left(C^{\prime} \mu\right)^{m} m^{-m / 2+l}\right\}
\end{aligned}
$$

Here, the constants $C, C^{\prime}$ are independent of $\rho, \mu$ and $m$.

Proof. The estimate (3.3) of Theorem 3.2 follows from Lemma 3.1 immediately. The estimate (3.2) is also easily checked:

$$
\begin{aligned}
&\left\|Q_{\mu}^{m}(x ; M)-Q_{\mu}^{m}(x ; \widetilde{M})\right\|_{C^{l}} \leq \sum_{|\alpha+\beta| \leq m}\left|C_{m, \alpha, \beta}\right| \mu^{n+|\alpha+\beta|} r_{1}^{|\alpha|} \\
&\|M-\widetilde{M}\|_{m} \sum_{i, j=1}^{N(m)}\left|C_{i j}^{\beta}\right|\left\|p_{i}^{m}\right\|\left\|p_{j}^{m}\right\|,
\end{aligned}
$$

where $r_{1}=\max |x|, \quad x \in \Omega$. Since each $C_{m, \alpha, \beta}$ is the coefficient of the expansion of $\delta_{\mu}^{m}(x-y)$, we obtain

$$
\sum_{|\alpha+\beta| \leq m}\left|C_{m, \alpha, \beta}\right| \mu^{n+|\alpha+\beta|} r_{1}^{|\alpha|} \leq(\sqrt{\pi})^{-n} \mu^{n} e^{n \mu^{2}\left(r_{1}+1\right)^{2}},
$$

which implies the estimate (3.2).

From Theorem 3.2 and Theorem 2.1, we have 
Corollary 3.3. Let $\Phi_{\rho}(t ; p, q)$ be the function of form (2.1). Then

$$
\begin{aligned}
\| \rho(x) & -Q_{\mu}^{m}\left(x ; \Phi_{\rho}(t ; \cdot, \cdot)\right) \|_{C^{l}\left(\mu^{-1 / 2}\right)} \\
\leq & C_{l}\left\{\|\rho\|_{C^{l+\sigma}} \mu^{-\sigma / 2}+(C \mu)^{m} m^{-m / 2+l}\right. \\
& \left.+e^{C^{\prime} \mu^{2}} e^{-C \lambda t} \max _{|\beta| \leq m ; i, j=1, \cdots, N(m)} \sum_{i, j=1}^{N(m)}\left|C_{i j}^{\beta}\right|\left\|p_{i}^{m}\right\|\left\|p_{j}^{m}\right\|\right\}
\end{aligned}
$$

for the constants $C, C^{\prime}$ are independent of $\rho, \mu$ and $m$.

Corollary 3.3 implies that we can reconstruct $\rho$ approximately via $\Phi_{\rho}(t ; \cdot, \cdot)$. Indeed, for any $\epsilon>0$ and compact set $D$ in $\Omega$, there exist $\mu, m$ and $t$ such that $\left\|\rho(x)-Q_{\mu}^{m}\left(x ; \Phi_{\rho}(t ; \cdot, \cdot)\right)\right\|_{C^{l}(D)}<\epsilon$.

Further analysis of the estimate in Corollary 3.3 involves an estimate of $\sum_{i, j=1}^{N(m)}\left|C_{i j}^{\beta}\right|\left\|p_{i}^{m}\right\|\left\|p_{j}^{m}\right\|$. Together with an optimal choice of the parameter $\mu$ this gives the final estimate which involves only $m$ and $t$.

Theorem 3.4. Let $\Phi_{\rho}(t ; p, q)$ be the functional introduced in Theorem 2.3 and $Q^{m}\left(x ; \Phi_{\rho}\right)$ - the polynomials defined by formula (3.1) with $M=\Phi_{\rho}$ and $\mu=m^{1 / 2}$. Then, there exist constants $C>0, c_{1}>0$ and $c_{2}>0$ such that

$$
\begin{aligned}
\| \rho(x)-Q^{m}(x & \left.; \Phi_{\rho}(t ; \cdot, \cdot)\right) \|_{C^{l}\left(m^{-1 / 4}\right)} \\
& \leq C\left\{\left(\|\rho\|_{C^{l+\sigma}}+1\right) m^{-\sigma / 4}+e^{c_{1} m-c_{2} \lambda_{0} t}\right\} .
\end{aligned}
$$

Proof. We employ the directional moments of order $q$ :

$$
X_{e}^{q}(x)=<x, e>^{q}, \quad e \in \mathbf{S}^{n-1},
$$

where $\mathbf{S}^{n-1}$ is the unit sphere in $\mathbf{R}^{n}$ and $<\cdot, \cdot>$ stands for the scalar product in $\mathbf{R}^{n}$. The goal of the proof is to find a representation

$$
x^{\alpha}=\sum_{\gamma=1}^{P(q)} c_{\gamma}^{\alpha} X_{e_{\gamma}}^{q}(x)
$$

and to estimate $\sum_{\gamma=1}^{P(q)}\left|c_{\gamma}^{\alpha}\right|,|\alpha|=q \leq m$.

Consider polynomials of the form $X_{e_{1}}^{q_{1}}(x) X_{e_{2}}^{q_{2}}(x)$ where $<e_{1}, e_{2}>=0$. Then (for details see $[\mathrm{K}-\mathrm{S}]$ )

$$
X_{e_{\phi}}^{q}(x)=\sum_{q_{1}+q_{2}=q} C_{q_{1}}^{q} \cos ^{q}(\phi) \tan ^{q_{1}}(\phi) X_{e_{1}}^{q_{1}}(x) X_{e_{2}}^{q_{2}}(x)
$$


where $e_{\phi}=e_{1} \cos (\phi)+e_{2} \sin (\phi)$ and $C_{q_{1}}^{q}$ are the binomial coefficients.

Equations (3.4) with $\phi=\phi_{1}, \cdots, \phi_{q+1}$ where $\tan \left(\phi_{i}\right) \neq \tan \left(\phi_{j}\right), \quad i \neq$ $j$ form a system of linear equations for the unknown $X_{e_{1}}^{q_{1}}(x) X_{e_{2}}^{q_{2}}(x)$. The corresponding matrix is essentially the Vandermonde matrix for $\tan \left(\phi_{i}\right), i=$ $1, \cdots, q+1$. In the following we take $\tan \left(\phi_{i}\right)=1+(i-1) / q$. Then

$$
X_{e_{1}}^{q_{1}}(x) X_{e_{2}}^{q_{2}}(x)=\sum_{i=1}^{q+1} \frac{1}{C_{q_{1}}^{q} \cos ^{q}\left(\phi_{i}\right)} \frac{\Delta_{q_{1}, i}}{\Delta} X_{e_{i}}^{q}(x),
$$

where $e_{i}=e_{\phi_{i}}$ and $\Delta, \Delta_{q_{1}, i}$ denote the determinant and $\left(q_{1}, i\right)$ minor of the Vandermonde matrix, correspondingly.

Denote by $\Delta_{q_{1}}(z)$ the determinant of the Vandermonde matrix with $z$ instead of $\tan \left(\phi_{q_{1}}\right)$;

$$
\Delta_{q_{1}}(z)=\sum_{j=1}^{q+1} \Delta_{q_{1}, j} z^{j-1}
$$

As, on the other hand,

$\Delta_{q_{1}}(z)=\Pi_{i<j ; i, j \neq q_{1}}\left(\tan \left(\phi_{i}\right)-\tan \left(\phi_{j}\right)\right) \Pi_{i<q_{1}}\left(z-\tan \left(\phi_{i}\right)\right) \Pi_{i>q_{1}}\left(\tan \left(\phi_{i}\right)-z\right)$,

we obtain that

$$
\frac{\Delta_{q_{1}, i}}{\Delta}=\frac{1}{2 \pi i} \int_{\Gamma} \frac{\Delta_{q_{1}}(z)}{\Delta} \frac{d z}{z^{i}}
$$

where $\Gamma$ is e.g. a circle of the radius 1 . But

$$
\frac{\Delta_{q_{1}}(z)}{\Delta}=\Pi_{i<q_{1}} \frac{\left(z-\tan \left(\phi_{i}\right)\right)}{\left(\tan \left(\phi_{q_{1}}\right)-\tan \left(\phi_{i}\right)\right)} \Pi_{i>q_{1}} \frac{\left(\tan \left(\phi_{i}\right)-z\right)}{\left(\tan \left(\phi_{i}\right)-\tan \left(\phi_{q_{1}}\right)\right)} .
$$

However, as $\tan \left(\phi_{i}\right)=1+(i-1) / q$

$$
\Pi_{i \neq q_{1}}\left|\left(\tan \left(\phi_{i}\right)-\tan \left(\phi_{q_{1}}\right)\right)\right| \geq\left(\frac{q !}{q^{q}}\right)^{2} \geq C^{-q} .
$$

Substitution of this estimate into (3.6) leads to the estimate

$$
\left|\frac{\Delta_{q_{1}, i}}{\Delta}\right| \leq C^{q}
$$

Returning to the estimate for $X_{e_{1}}^{q_{1}}(x) X_{e_{2}}^{q_{2}}(x), q_{1}+q_{2}=q$ we use the estimate $\cos \left(\phi_{i}\right) \geq 5^{-1 / 2}$. Hence equations (3.5), (3.7) yield that

$$
X_{e_{1}}^{q_{1}}(x) X_{e_{2}}^{q_{2}}(x)=\sum_{i=1, \cdots, q+1} c_{q_{1}, i} X_{e_{i}}^{q}(x) ; \quad\left|c_{q_{1}, i}\right| \leq C^{q} .
$$


Let us consider $x^{\alpha}=x_{1}^{\alpha_{1}} \cdots x_{n}^{\alpha_{n}}$. By induction, it is easy to show that formula (3.8) yields the representation

$$
x^{\alpha}=\sum_{i=1}^{P(q)} c_{i}^{\alpha} X_{e_{i}}^{q}(x), \quad|\alpha|=q,
$$

where

$$
P(q) \leq(1+q)^{n}, \quad\left|c_{i}^{\alpha}\right| \leq C^{q}
$$

Furthermore

$$
X_{e}^{q}=2^{-q}\left(Z_{e}+\bar{Z}_{e}\right)^{q}=2^{-q} \sum_{q_{1}=0}^{q} D_{q_{1}}^{q} Z_{e}^{q_{1}} \bar{Z}_{e}^{q-q_{1}},
$$

where $Z_{e}=<x, e>+i<x, e^{\prime}>$ for an arbitrary $e^{\prime}$ such that $<e, e^{\prime}>=0$. Then

$$
\begin{gathered}
x^{\alpha}=\sum_{i=1}^{P(q)} c_{i}^{\alpha} X_{e_{i}}^{q}=\sum_{i=1}^{P(q)} c_{i}^{\alpha} 2^{-q} \sum_{q_{1}=0}^{q} D_{q_{1}}^{q} Z_{e_{i}}^{q_{1}} \bar{Z}_{e_{i}}^{q-q_{1}} \\
=\sum_{j, k=1}^{N(q)} c_{j, k}^{\alpha} p_{j}(x) \bar{p}_{k}(x),
\end{gathered}
$$

Here $p_{j}$ are harmonic polynomials of the form: $p_{j}(x)=Z_{e_{i}}^{q_{1}}(x)$ with $q_{1} \leq q$ and $p_{k}(x)=Z_{e_{i}}^{q-q_{1}}(x)$. Moreover, as $\sum_{q_{1}=0}^{q} D_{q_{1}}^{q}=2^{q}$

$$
\sum_{j, k}^{N(q)}\left|c_{j, k}^{\alpha}\right|=\sum_{i=1}^{P(q)}\left|c_{i}^{\alpha}\right| 2^{-q} \sum_{q_{1}=0}^{q}\left|D_{q_{1}}^{q}\right| \leq q^{n} C^{q} \leq C_{n}^{q} .
$$

As $\left\|p_{j}\right\|\left\|p_{k}\right\| \leq V(\Omega)\left(1+r_{1}\right)^{q}$, where $V(\Omega)$ is the volume of $\Omega$, the above estimate together with Theorem 3.2 and Corollary 3.3 give rise to the following estimate:

$$
\begin{array}{r}
\left\|\rho(x)-Q_{\mu}^{m}\left(x ; \Phi_{\rho}(t ; \cdot, \cdot)\right)\right\|_{C^{l}\left(\mu^{-1 / 2}\right)} \leq C\left\{\|\rho\|_{C^{l+\sigma}} \mu^{-\sigma / 2}\right. \\
\left.+(C \mu)^{m} m^{-m / 2+l}+e^{C^{\prime} \mu^{2}-C \lambda_{0} t} C^{m}\right\}
\end{array}
$$

Thus, inserting $\mu=m^{1 / 2}$ into the above inequality, we obtain the estimate in Theorem 3.4 .

Analysing the proof of Theorem 3.4 we obtain also the following stability estimate which will be used in $\S 4$ : 
Lemma 3.5. Let $M^{m}, \widetilde{M}^{m}$ be bilinear forms from $H P^{m}$ to $C^{0}(0,2)$. Let $Q_{\mu}^{m}(x ; M)$ and $Q_{\mu}^{m}(x ; \widetilde{M})$ be given by formula (3.1). Then

$$
\left\|Q_{\mu}^{m}(x ; M)-Q_{\mu}^{m}(x ; \widetilde{M})\right\|_{C^{l}} \leq C_{l}\|M-\widetilde{M}\|_{m} e^{c^{\prime} \mu^{2}} .
$$

\section{$\S 4$. Analytic estimates and stability}

In the analysis of $\S 3$ we have not used the fact that $\Phi_{\rho}(t ; \cdot, \cdot)$ is an analytic function when $\Re(t)>1$ which makes possible to improve the estimates of Corollary 3.3 and Theorem 3.4 and to obtain some further stability results for the considered inverse problem.

We start with stability estimates.

Lemma 4.1. Let $\rho, \widetilde{\rho}$ satisfy conditions (1.2) and

$$
\left\|\Phi_{\rho}(t ; p, q)-\Phi_{\rho}(t ; p, q)\right\|_{C^{0}(0,2)} \leq \epsilon\|p\|\|q\| \quad p, q \in H P^{m}
$$

then

$$
\left|M_{\rho}(p, q)-M_{\rho}(p, q)\right| \leq C e^{C \lambda^{1 / 2}|\lg \epsilon|^{1 / 2}}\|p\|\|q\| .
$$

Proof. Let $z=\frac{t-2}{t}$ be a conformal map of the halfplane $\Re(t)>1$ onto the unit disk $|z|<1$. Consider the function

$$
f(z)=\Phi_{\rho}(t ; p, q)-\Phi_{\rho}(t ; p, q), \quad z=z(t) .
$$

which is analytic in the unit disk. Moreover,

$$
\begin{gathered}
|f(z)| \leq 2 \rho_{2}\|p\|\|q\|, \quad|z| \leq 1 \\
|f(z)| \leq \epsilon\|p\|\|q\|, \quad-1 \leq z \leq 0 .
\end{gathered}
$$

By Milloux Theorem (see e.g [G; Ch.VIII, §4, Th. 6]) these estimates imply that when $z=1-\zeta, \Im(z)=0$,

$$
|f(z)| \leq 2 \rho_{2}\|p\|\|q\| \epsilon^{\zeta / \pi} .
$$

Taking $\zeta=2 / t$ we see that

$$
\left|\Phi_{\rho}(t ; p, q)-\Phi_{\rho}(t ; p, q)\right| \leq 2 \rho_{2}\|p\| \||| \mid \epsilon^{2 / \pi t} .
$$

This estimate together with Theorem 2.1 (iii) where $t=C\left(\frac{|\lg \epsilon|}{\lambda_{0}}\right)^{1 / 2}$ prove the statement.

The Proof is complete.

Lemma 4.1 together with Theorem 3.2 (iii) and Lemma 3.4 lead to the following stability result: 
Theorem 4.2. Let $\rho, \widetilde{\rho}$ satisfy conditions (1.2) and

$$
\left\|\Phi_{\rho}(t ; p, q)-\Phi_{\rho}(t ; p, q)\right\|_{C^{0}(0,2)} \leq \epsilon\|p\|\|q\| ; \quad p, q \in H P^{m} .
$$

Then

$$
\begin{aligned}
\| \rho(x)- & \widetilde{\rho}(x) \|_{C^{l}\left(\mu^{-1 / 2}\right)} \\
\leq & C\left\{\|\rho\|_{C^{l+\sigma}} \mu^{-\sigma / 2}+e^{C \mu^{2}}(C \mu)^{m} m^{-m / 2}\right. \\
& \left.+e^{C \mu^{2}}(C \mu)^{m} e^{\left\{-C \lambda^{1 / 2}|\lg \epsilon|^{1 / 2}\right\}}\right\}
\end{aligned}
$$

Proof. Obviously

$$
\begin{aligned}
& \|\rho-\tilde{\rho}\| \leq\left\|\rho-Q_{\mu}^{m}\left(x, M_{\rho}\right)\right\|+ \\
& \quad+\left\|\tilde{\rho}-Q_{\mu}^{m}\left(x, M_{\tilde{\rho}}\right)\right\|+\left\|Q_{\mu}^{m}\left(x, M_{\rho}\right)-Q_{\mu}^{m}\left(x, M_{\tilde{\rho}}\right)\right\| .
\end{aligned}
$$

The first two terms in rhs of (4.2) may be estimated by means of (3.3). To estimate the third term we use the relation (4.1) together with Lemma 3.5 with $M_{\rho}, M_{\tilde{\rho}}$ instead of $M, \widetilde{M}$.

The above estimate may be simplified if we take $\mu=e^{-a} m^{1 / 2}$ where $a$ is a sufficiently large positive number which depends upon $\Omega, \rho_{1}, \rho_{2}$. Then

$$
\begin{gathered}
\|\rho(x)-\widetilde{\rho}(x)\|_{C^{l}\left(m^{1 / 4}\right)} \\
\leq C\left\{\|\rho\|_{C^{l+\sigma}} m^{-\sigma / 4}+e^{-C m}+\exp \left(C_{1} m-C_{2} \lambda^{1 / 2}|\lg \epsilon|^{1 / 2}\right)\right\} .
\end{gathered}
$$

Our next goal is to improve the reconstruction procedure described in $\S 3$ for the case when $\Phi_{\rho}(t ; \cdot, \cdot)$ is known with some error. Let $\Psi_{\epsilon}(t ; p, q), p, q \in$ $H P^{m}, t \in[0,2]$ is such that

$$
\left\|\Phi_{\rho}(\cdot ; p, q)-\Psi_{\epsilon}(\cdot ; p, q)\right\|_{C^{0}(0,2)} \leq \epsilon\|p\|\|q\| .
$$

Let

$$
w(t)=\frac{\left\{1-(t-1)^{2}\right\}^{1 / 2}-i}{\left\{1-(t-1)^{2}\right\}^{1 / 2}+i}
$$

be a conformal map of the halfplane $\Re(t)>1$ with a slit along the interval $(1,2)$ onto the unit disk $|w|<1$. Then the slit is transformed onto the left semicircle, $|w|=1, \Re(w)<0$ and the line $\Re(t)=1$ onto the right semicircle $|w|=1, \Re(w)>0$. The function $f(w ; p, q)=\Phi_{\rho}(t ; p, q), w=w(t)$ is analytic in the disk. 
Let $f_{\epsilon}(w ; p, q)=\Psi_{\epsilon}(t ; p, q), t \in(1,2), w=w(t)$. Then $f_{\epsilon}$ is defined on the left semicircle and

$$
\left|f(w)-f_{\epsilon}(w)\right| \leq \epsilon\|p\|\|q\|
$$

Consider the domain $\mathcal{O}$ (see Fig.1) obtained as the intersection of the disk and the sector of the angle $\pi / 2$ with its vortex in the point $(1,0)$ which is symmetric with respect to the real axis.

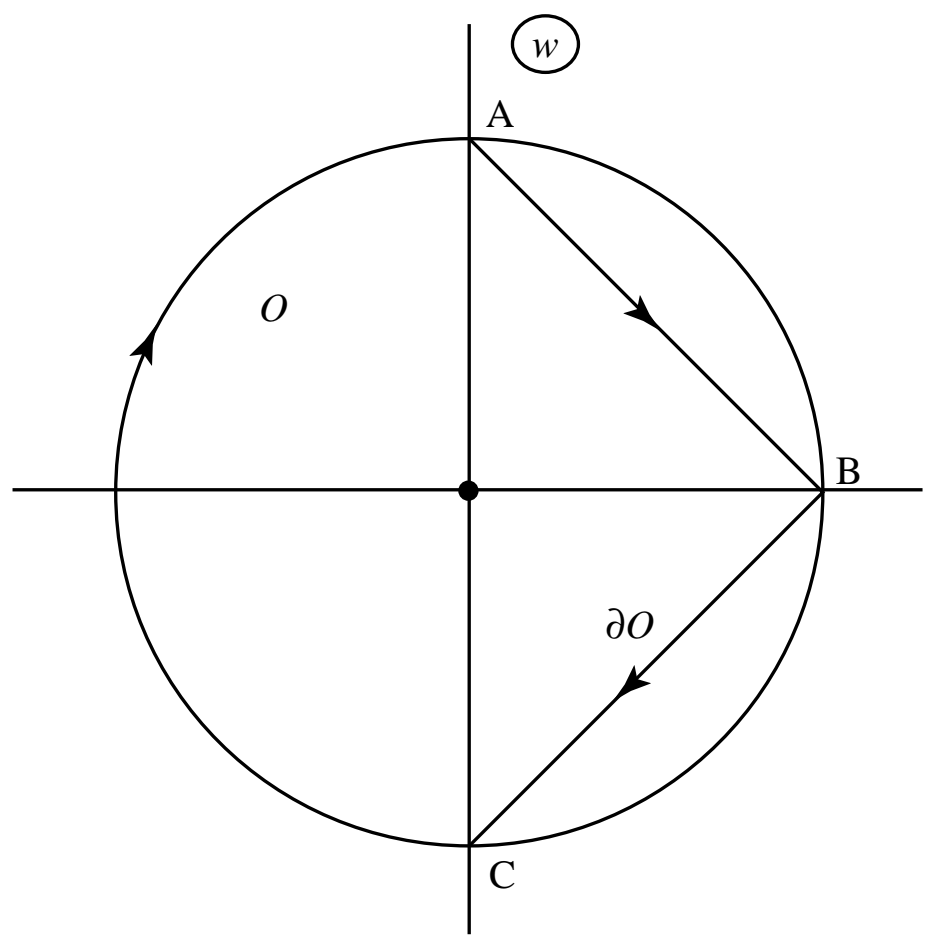

For the quasianalytic continuation of $f_{\epsilon}(w)$ we use the construction suggested in [L ] which is based upon the Carleman lemma [Car]. Let

$$
\sigma_{\epsilon}=\frac{1}{t^{2}} \log \frac{C t}{\epsilon}
$$

with some $t \geq 2$. We define $\tilde{f}_{\epsilon}^{t}(z)$ by the following formula:

$$
\tilde{f}_{\epsilon}^{t}(z)=\frac{e^{-\sigma_{\epsilon}}}{2 \pi i} \int_{\frac{\pi}{2}}^{\frac{3 \pi}{2}} f_{\epsilon}(w) \exp \left\{\sigma_{\epsilon}\left(\frac{w-1}{z-1}\right)^{2}\right\} \frac{i w d \phi}{z-w}, \quad w=e^{i \phi}
$$


Theorem 4.3. Let $\tilde{f}_{\epsilon}^{t}(z)$ be given by (4.4) where $f_{\epsilon}$ satisfies (4.3). Then

$$
\begin{gathered}
\left|M_{\rho}(p, q)-\tilde{f}_{\epsilon}^{t}(1-2 / t)\right| \\
\leq C\|p\|\|q\|\left\{t \epsilon^{\frac{1}{t^{2}}}+e^{-C \lambda t}\right\}
\end{gathered}
$$

Proof. Consider $\tilde{f}_{\epsilon}^{t}(z)-f(z)$. Using the Cauchy formula for $f^{t}(z)=$ $f(z) \exp \left(\frac{\sigma_{\epsilon}(z-1)^{2} t^{2}}{4}\right)$ we see that

$$
f(1-2 / t)=\frac{e^{\sigma_{\epsilon}}}{2 \pi i} \int_{\partial \mathcal{O}} f^{t}(z) \frac{d z}{1-2 / t-z}
$$

where the contour $\partial \mathcal{O}$ consists of the left semicircle $\Re(z)<0$ and the broken line $A B C$ (see Fig. 1). Hence

$$
\begin{gathered}
\left|\tilde{f}_{\epsilon}^{t}(1-2 / t)-f(1-2 / t)\right| \\
\leq \frac{e^{\sigma_{\epsilon}}}{2 \pi} \int_{\frac{\pi}{2}}^{\frac{3 \pi}{2}}\left|\tilde{f}_{\epsilon}(z)-f(z)\right| \exp \left(\frac{\sigma_{\epsilon} t^{2}}{4} \Re(z-1)^{2}\right)\left|\frac{d z}{1-2 / t-z}\right| \\
+\frac{e^{\sigma_{\epsilon}}}{2 \pi} \int_{A B C}|f(z)| \exp \left(\frac{\sigma_{\epsilon} t^{2}}{4} \Re(z-1)^{2}\right)\left|\frac{d z}{1-2 / t-z}\right|= \\
I_{1}+I_{2},
\end{gathered}
$$

where $z=e^{i \phi}, \quad \phi \in\left(\frac{\pi}{2}, \frac{3 \pi}{2}\right)$ in the first integral $I_{1}$. However, $\Re(z-1)^{2} \leq 2$ and $|1-2 / t-z| \geq 1$ when $z=e^{i \phi}, \phi \in\left(\frac{\pi}{2}, \frac{3 \pi}{2}\right)$. Hence inequality (4.3) yields the following estimate for $I_{1}$ :

$$
I_{1} \leq C \epsilon\|p\|\|q\| e^{\sigma_{\epsilon}\left(t^{2}-1\right)} \leq C t \epsilon^{\frac{1}{t^{2}}}\|p\|\|q\|,
$$

where the last estimate follows from the definition of $\sigma_{\epsilon}$.

To estimate $I_{2}$ we use the fact that $\Re\left\{(z-1)^{2}\right\}=0$ and $|1-2 / t-z| \geq \frac{\sqrt{2}}{t}$ on $A B C$. Taking into account estimate (i) of Theorem 2.1 we see that

$$
I_{2} \leq C t\|p\|\|q\| e^{\sigma_{\epsilon}} \leq C t \epsilon \frac{1}{t^{2}}\|p\|\|q\| .
$$

Clearly $\left|w(t)-\left(1-\frac{2}{t}\right)\right| \leq \frac{C}{t^{2}}$ so that $1-\frac{2}{t}=w(\tilde{t})$, where $|t-\tilde{t}| \leq C$. Taking into account Theorem 2.1(iii) we see that

$$
\left|f(1-2 / t)-M_{\rho}\right|=\left|\Phi_{\rho}(\tilde{t} ; p, q)-M_{\rho}(p . q)\right| \leq C e^{-C^{\prime} \lambda t}\|p\|\|q\| .
$$


Equation (4.5) follows from (4.6)- (4.9).

The proof is complete.

Remark. The considerations leading to (4.8) is a special case of the results obtained by M.Lavrent'ev (see [L; Ch.I, Pr.III]).

Let $M_{\rho}^{\epsilon}(p, q)=\tilde{f}_{\epsilon}^{t}(1-2 / t)$, where $t=|\log \epsilon|^{1 / 3} \lambda_{0}^{-1 / 3}$. Then estimate (4.9) implies that

$$
\left|M_{\rho}(p, q)-M_{\rho}^{\epsilon}(p, q)\right| \leq C|\log \epsilon|^{1 / 3} e^{-\lambda_{0}^{2 / 3}|\log \epsilon|^{1 / 3}}
$$

Summarising the previous considerations we come to the following

Theorem 4.4. Let $\Psi_{\epsilon}: H P^{m} \times H P^{m} \rightarrow C^{0}(0,2)$ be a bilinear form which is $\epsilon$-close to $\Phi_{\rho}$. Then

$$
\begin{gathered}
\left\|\rho(\cdot)-Q_{\mu}^{m}\left(\cdot ; M_{\rho}^{\epsilon}\right)\right\|_{C^{l}\left(\mu^{-1 / 2}\right)} \\
\leq C\left\{\|\rho\|_{C^{l+\sigma}} \mu^{-\sigma / 2}+e^{r_{0}^{2} \mu^{2}} \mu^{n}\left(\mu r_{0}\right)^{m} /\left(\frac{m}{2}\right) !\right. \\
\left.+|\log \epsilon|^{1 / 3} e^{n^{2}\left(r_{1}+1\right)^{2} \mu^{2}}\left[m \mu^{2}\right]^{n}\left[5^{3 n / 2}\left(1+r_{1}\right)^{2}\right]^{m} e^{-\lambda_{0}^{2 / 3}|\log \epsilon|^{1 / 3}}\right\} .
\end{gathered}
$$

In particular when $\mu=m^{1 / 2}$ we have

$$
\begin{gathered}
\left\|\rho(\cdot)-Q_{m^{1 / 2}}^{m}\left(\cdot ; M_{\rho}^{\epsilon}\right)\right\|_{C^{l}\left(m^{-1 / 4}\right)} \\
\leq C\left(1+\|\rho\|_{C^{l+\sigma}}\right)\left(m^{-\sigma / 4}+\exp \left(C m-C^{\prime} \lambda_{0}^{2 / 3}|\log \epsilon|^{1 / 3}\right)\right) .
\end{gathered}
$$

\section{References}

[Cal] Calderon A. On an inverse boundary value problem, in: Semin. Num. Anal. and Its Appl. to Cont. Phys., Rio de Janeiro (1980), p. 65-73.

[Car] Carleman T. Les Fonctions QuasiAnalitiques, Paris (1926).

[G] Goluzin G.M. Geometric Theory of Functions of a Complex Variable, Transl. of Math. Monograph., v. 26 (1969), AMS. 676 pp.

[Is] Isakov V. Inverse Problems for Partial Differential Equations, Appl. Math. Sci,. v. 127 (1998), Springer. 284 pp. 
[K-P] Kurylev Y. and Peat K.S. Hausdorff moments in two-dimensional inverse acoustic problem, Inv. Probl., v. 13 (1997), p. 1363-1377.

[K-S] Kurylev Y. and Starkov A. Directional moments in the acoustic inverse problem, in: Inverse Probl. in Wave Propag. (ed. Chavent G et al), IMA Vol. in Math. and Its appl, v.90 (1997), p.295-324. Springer.

[L] Lavrent'ev M.M. Cauchy problem for the laplace equation, Izv. Akad. Nauk SSSR, Ser. Matem. (in Russian), v.20 (1956), p.819-842.

[S-U] Sylvester J. and Uhlmann G. A global uniqueness theorem for an inverse boundary value problem, ann. Math., v.125 (1987), p. 153-169. 